\title{
WHITE-LIGHT RECONSTRUCTION OF HOLOGRAPHIC IMAGES USING THE LIPPMANN-BRAGG DIFFRACTION EFFECT*
}

\author{
G.W.STROKE and A. E. LABEYRIE \\ The University of Michigan, Ann Arbor Michigan
}

Received 27 January 1966

\begin{abstract}
An extension of the 1962 Denisyuk method to record reflection holograms in Lippmann emulsions has permitted us to reconstruct monochromatic images with white light (e.g.sun) and demonstrate possibilities of simulating three-dimensional gratings for crystallographic studies.
\end{abstract}

Fig. 1a shows an arrangement which we have used for recording holograms of two- or threedimensional objects in such a way that illumination of the hologram with ordinary sun-light (or other white light, e.g. from a flash light), as in fig. 1b will produce a 'single-color' image of the object by wavefront reconstruction. An example of a reconstruction of the image of a grasshopper which we have obtained with sun-light illumination of such a hologram is shown in fig. 2. The original object in this case was a $24 \times 36 \mathrm{~mm}^{2}$ Kodachrome transparency placed at about 1 inch from the photographic plate (along the $z$-direction), so as to produce a reflection version of a 'projection' hologram [1], by recording in $6328 \AA$ laser light. Reconstructions with three-dimensional objects, both diffusing and speculary reflecting have been equally successful.

The physical principles of holographic imaging with the method illustrated in fig. 1 may be readily given in simple terms. Basically the 'singlecolor' selection in the reconstruction with white light may be attributed to a multilayer interference effect, resulting from the stratifications of the emulsion caused in the recording fig. 1a by the interference between the reference field and the field scattered by the object, very much like in the original Lippmann color photography method [2], first described in 1894. The spacing of the stratifications (along the $z$-direction) in the recording is $\frac{1}{2} \lambda$ in case where both fields are plane waves incident normally on the plate. In the case of scattering by an arbitrary object (fig. 1a), the multilayer stratification maxima are locally displaced along $z$, according to the local values

* Parts of this work were first publicly presented by G.W. Stroke on 5 January 1966 in the board room at the National Science Foundation, Washington, D.C., by special invitation from Dr. John M. Ide, NSF Division Director for Engineering.

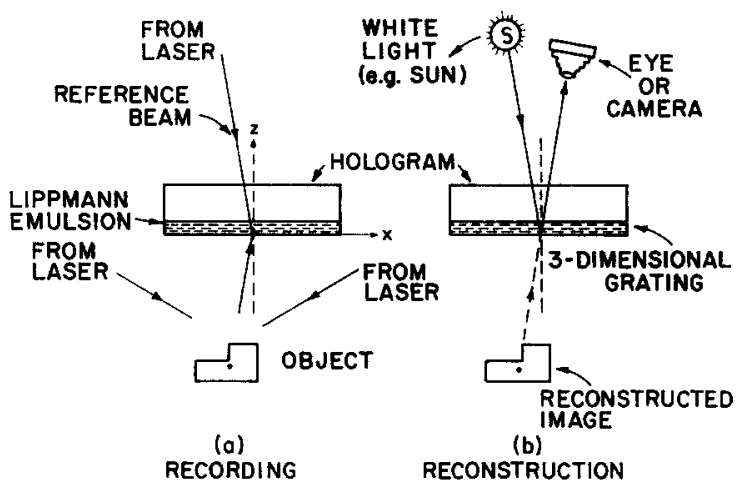

Fig. 1a. Recording (in laser light) of crystal (grating)like hologram capable of $b$. reconstructing single-color images upon white-light illumination, using a LippmannBragg diffraction effect.

of the phase of the resultant scattered electricfield vector (as measured with respect to the reference field). In addition, the resultant local intensity modulation of the processed photographic emulsion (in depth, say along $z$, for a given $x$-coordinate in the plane of the hologram) is determined by the resultant magnitude of the scattered field vector.

In the resconstruction (fig. 1b) white-light illumination of the hologram from the reference beam side produces, just like from a diffracting crystal, one spectrally selected single-color 'reconstructed wave', capable of forming an image by wavefront reconstruction, according to the principles first described by Gabor in 1948 [3]. In the 'reconstructed wave', (1) the local phase modulations are determined by the local displacements of the reflecting multiplayer stratifications, (2) the local amplitude modulation is determined by the local intensity modulation of the hologram, and (3) the color selection is obtained by Bragg diffraction from the grating- 
like stratifications. (The spacing of the stratifications may be suitably varied by omitting photographic fixing and other techniques $[6,7]$.

A basic theory of this type of reflection hologram recording was first given by Yu. N. Denisyuk in 1962 [4], for the case when the object was actually illuminated through the hologram, rather than with a separated beam (split before the incidence on the hologram, as in fig. 1a). An experiment with mirrors used as objects and recording with mercury light was also described by Denisyuk, but no photographic results were shown, presumably because of the well known difficulties of recording good holograms before the advent of lasers [3]. The readily obtained very good result which we show here was obtained with sun-light reconstruction from a hologram recorded in $6328 \AA$ laser light. (Equally good and readily observable images are obtained with very sufficient brightness with the light from a small pocket flash light.)(It may be of interest to note that an arrangement similar to that which we use for the recording of Lippmann-Bragg reflection holograms has been described in ref. 5 . for the purpose of demonstrating the possibility of using "inverted reference beam" illumination in the recording of 'monochromatic transmission holograms', such as those in general use heretofore, and not for the purpose of recording the 'white-light reflection holograms' which we describe here. The inverted reference-beam illumination in the recording of transmission holograms has the important advantage, pointed out in ref:5., of permitting one to place the reference mirror close to the laser source, rather than next to the distant object, when a sufficient coherence length [1] in the laser is available. Reflection images from conventional 'transmission' holograms upon illumination with monochromatic laser light have also been widely observed. In a general way all methods of holography are based on the original work by Gabor [3].)

The theoretical description of the white-light reflection holography process which we describe here may be readly given in the form of a 'modulated Lippmann-Bragg reflection-grating' formulation. Details will be given in a more extensive publication [6]. For the case of a scattered field describable by $A(x) \exp i \varphi(x)$ on the hologram surface $(x$-plane), say upon normal incidence along the $z$-direction, and with the reference field also incident along the $z$-direction, the intensity recorded in the hologram emulsion may be written as $I(x, z) \propto 1+A(x) \cos \{2 k z+\varphi(x)\}$, where $k=2 \pi / \lambda$ and $\lambda$ is the recording wavelength. Illumination with a 'plane' white-light wave reconstructs the field $A(x) \exp i \varphi(x)$ in a single wavelength by a holographic Lippmann-Bragg diffraction process from the three-dimensional

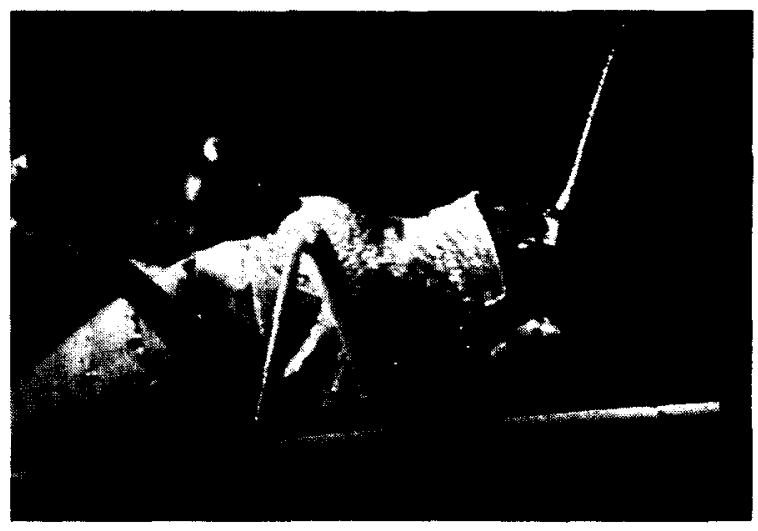

Fig. 2. Image of a grasshopper reconstructed with sunlight illumination of a hologram according to fig. $1 \mathrm{~b}$. (The original 'object' in this case was a $24 \times 36 \mathrm{~mm}^{2}$ Kodachrome transparency, placed at about $25 \mathrm{~mm}$ in front of the hologram which was recorded in $6328 \AA$ laser light, see text.) Very bright, sharp images were obtained by reconstruction from the same hologram upon illumination with only the light from an ordinary pocket flash light.

grating described by the equation.

(It may be of interest to note that particularly brilliant reconstructions are obtained in this manner by recording the hologram in an arrangement where the scattered field is brought to focus, say with a lens, at some distance behind the hologram, on the reference-beam side, after the illuminating beam has been scattered by the object, e.g. the transparency used in the recording of the hologram for fig. 2. Among many other experimental refinements which we have investigated, we have also found most useful the techniques of treating Lippmann emulsions described by Ives [ 7 ] in 1908, especially in controlling the spacing of the elementary stratifications.)

In our ref. 6 . we shall also further discuss extensions of our method of 'reflection holography' to the generation of three-dimensional crystallike gratings in photographic emulsions, which may be of a particular interest for the purpose of simulating crystallographic lattices for diffraction studies in the visible-light domain, and possibly for simplifying or aiding some aspects of X-ray image synthesis, e.g. in crystallography and protein structure investigations. Applications to interferometry, spectroscopy as well as to color holography, among others, have also appeared to be possible.

We wish to especially acknowledge most fruitful conversations with Gilbert B. Devey which were at the origin of generating our interest in investigating possibilities of 'white-light holography', such as that described here. We also wish to acknowledge the interest and fruitful conversations with several colleagues, notably 
with R.C. Restrick. We are most grateful for a most generous grant from the National Science Foundation in support of major parts of our research.

\section{References}

1. G.W. Stroke, An introduction to coherent optics and holography (Academic Press Inc., New York and London, 1966), 270 pages (including reprints of the three original wavefront-reconstruction papers by D. Gabor).
2. G. Lippmann, J. de Physique 3 (1894) 97.

3. D.Gabor, Nature, 161 (1948) 777.

4. Yu. N.Denisyuk, Soviet Physics-Doklady, 7 (1962) 543.

5. A.S.Hoffman, J.G.Doidge and D.G.Mooney, J. Opt. Soc. Am. 55 (1965) 1559.

6. A.E. Labeyrie and G.W.Stroke, Optica Acta (to be published)

7. H.E.Ives, Astrophys.J. 27 (1908) 325.

\title{
OBSERVATION OF SOUND WAVES IN AFTERGLOW PLASMAS*
} BY MEANS OF TONKS-DATTNER RESONANCES

\author{
K. J. NYGAARD \\ Sperry Rand Research Center, Sudbury, Massachusetts
}

Received 22 January 1966

\begin{abstract}
The electrical breakdown of a gas by a high voltage pulse will excite various sound waves. The propagation of an axial shock wave and a radial standing sound wave in afterglow plasmas has been studied by means of Tonks-Dattner resonances.
\end{abstract}

Recently, we have been using the electroacoustic Tonks-Dattner resonances to measure electron densities [1] and electron temperature transients [2] in noble-gas afterglow plasmas. During these investigations, we also observed perturbations on the Tonks-Dattner resonance curves due to sound waves in the neutral gas.

Other investigators [3] have reported on a standing sound wave, or a shock with Mach number very close to unity, following the breakdown of the gas in a discharge tube with a rectangular cross section. They detected the sound wave in the partially ionized gas by means of the changes that were produced in the transmission of a microwave signal and in the intensity of the recombination light. By means of the new TonksDattner resonance technique, we have discovered a radial sound wave following the electrical breakdown of the gas enclosed in a cylindrical discharge tube. The breakdown pulse also produced a shock wave that traveled parallel to the tube axis.

A schematic drawing of the hot filament discharge tube is shown in fig. 2. For simplicity,

*This work was supported in part by the Air Force Cambridge Research Laboratory, Office of Aerospace Research, under Contract No. AF19(628) -4183 . we have excluded all electronic equipment. A microwave signal was applied to the strip line, which could slide along the tube. The dipolar electric field between the arms of the strip line will excite local electroacoustic resonances at certain electron densities. The excitation and detection of these resonances is well known from the literature [4]. The microwave signal that is reflected from the plasma carries with it information pertaining to the Tonks-Dattner resonances as seen in fig. 1. This is an enlarged picture showing only the first resonance. Of particular interest here is the modulation of the resonance curve. Higher order resonances exhibit a similar modulation. The amplitude of this modulation was found to increase with the electrical energy dissipated in the tube. Therefore, in the following measurements, we used maximum available breakdown energies, which were of the order of 0.1 joule. The period $\tau_{0}$ of the modulation was found to be independent of neutral gas pressures from 1-10 Torr and independent of exciting frequencies between 500 and $1800 \mathrm{Mhz}$. We also found that the period did not depend on the position of the strip line along the tube. Furthermore, microwave heating of the electrons did not change $\tau_{0}$. Finally, the modulation was very weakly damped, since it could be observed as late as $2.5 \mathrm{msec}$ after breakdown. 